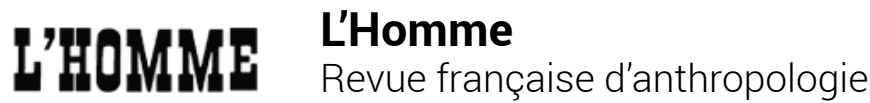

163 | juillet-sptembre 2002

De la légende au mythe. Parole, langue et pensée

\section{Entre recherche agronomique et questionnement éthique, l'animal de rente}

\section{Valentin Pelosse}

\section{(2) OpenEdition \\ 1 Journals}

\section{Édition électronique}

URL : http://journals.openedition.org/lhomme/12201

DOI : 10.4000//homme.12201

ISSN : 1953-8103

Éditeur

Éditions de l'EHESS

\section{Édition imprimée}

Date de publication : 21 juin 2002

Pagination : 217-228

ISBN : 2-7132-1771-7

ISSN : 0439-4216

\section{Référence électronique}

Valentin Pelosse, «Entre recherche agronomique et questionnement éthique, l'animal de rente »,

L'Homme [En ligne], 163 | juillet-sptembre 2002, mis en ligne le 03 juillet 2007, consulté le 22

septembre 2020. URL : http://journals.openedition.org//homme/12201 ; DOI : https://doi.org/10.4000/ Ihomme.12201 


\section{Entre recherche agronomique et questionnement éthique, l'animal de rente}

\author{
Come le pecorelle escon del chiuso \\ a una, a due, a tre, e l'altre stanno \\ timidette atterrando l'occhio e 'l muso, \\ e ciò che fa la prima, e l'altre fanno, \\ addossandosi a lei, s'ella s'arresta, \\ semplici e quete, e lo 'mperché non sanno; \\ sí vid' io muovere a venir la testa \\ di quella mandra fortunata allota, \\ pudica in faccia e ne l'andare onesta.
}

\begin{abstract}
Valentin Pelosse
"Comme les brebis sortent de leur étable, une, puis deux, puis trois, et les autres restent, timides, les yeux et le museau baissés,

et les autres font ce que fait la première, se serrant derrière elle, si elle s'arrête, simples et calmes, et ne savent le pourquoi; c'est ainsi que je vis bouger et venir alors la tête du troupeau fortuné, le visage pudique et la démarche honnête.»

Dante, Le Purgatoire, III, 78-87
\end{abstract}

$\mathrm{N}$

OUS ALLONS parler de viande sous cellophane. L'agronomie au sens moderne de la discipline émerge au milieu du XIX siècle en tant que volonté de se démarquer d'un empirisme agricole plus ou moins raisonné, comme projet de scientifisation de l'agriculture et d'imitation de la gestion industrielle. Créé en 1946, l'Institut national de la recherche agronomique (INRA) devient le protagoniste scientifique qui œuvre en amont de l'édification du puissant complexe agro-alimentaire français dans le cadre de la "politique agricole commune " européenne (PAC). L'efficacité technique et les succès économiques ont leur revers. La diffusion des innovations de la recherche grâce aux courroies de transmission que sont les organisations professionnelles agricoles entraîne des pratiques agro-pastorales intensives qui dégradent l'environnement naturel. Située en amont des filières animales - entendons l'ensemble des acteurs socioéconomiques impliqués dans une chaîne de production, la filière bovine, ou porcine, ou avicole, celle du lait, etc. -, la recherche zootechnique a été prioritairement orientée en faveur des systèmes d'élevage intensif confiné. Ceux-ci nécessitent la claustration et la contention à vie (brève) d'énormes effectifs d'animaux de rente dans des espaces exigus, au point de ne plus permettre aux bêtes de se mouvoir normalement en fonction des besoins comportementaux élémentaires de l'espèce. La bonne santé de l'animal, condition d'une bonne rentabilité, peut sous-entendre un bien-être aussi long1. Dante, La divine comédie. Texte original. Traduction, introduction et notes par Jacqueline Risset, Paris, Garnier-Flammarion, 1992 ( Bilingue»).

À propos de Florence Burgat, avec la collaboration de Robert Dantzer, Les Animaux d'élevage ont-ils droit au bien-être? Paris, INRA-Éditions, 2001 (avec les contributions de Françoise Armengaud, Antoine Garapon, Jean-Yves Goffi, Marie-Angèle Hermitte, Catherine \& Raphaël Larrère, Jocelyne Porcher) ; et de Élisabeth de Fontenay, Le Silence des bêtes. La philosophie à l'épreuve de l'animalité, Paris, Fayard, 1998. 
temps que les "animaux de ferme " sont élevés libres de se mouvoir. La généralisation des élevages hors-sol dans la deuxième moitié du XXe siècle en réponse à une logique économique de fabrication au moindre coût de produits animaux standardisés a disqualifié ces glissements sémantiques. L'élevage en batterie évoque pour le profane un univers concentrationnaire à l'usage des bêtes. Dès les années 1970, des associations de protection mènent campagne contre les souffrances qu'infligent aux animaux les méthodes industrielles d'élevage. Des modifications de la réglementation intéressant les conditions de transport, de manutention, d'abattage s'ensuivent, législation plus ou moins respectée. L'INRA lance en avril 1996 un appel d'offres pour financer des recherches à caractère pluridisciplinaire sur les conditions d'élevage des animaux de rente. Il fallait se conformer aux nouvelles directives européennes. On pose (p. 106) qu’à la conception purement utilitaire de l'animal d'élevage s'est substituée une vision inspirée par des "considérations éthiques et philosophiques" qui conferent à l'animal «le statut d'être vivant et sensible auquel il convient non seulement d'éviter toute souffrance inutile, mais également d'assurer confort et bien-être» ("Action incitative programmée ", 1996-1997). Le bien-être... l'intention était ambitieuse. Une philosophe a été recrutée. Les contraintes d'une recherche finalisée au sein d'une institution n'offrent pas que des inconvénients pour la réflexion philosophique si elles permettent une synergie. Florence Burgat a bénéficié du bilan scientifique établi par Robert Dantzer, vétérinaire neurobiologiste à l'INRA, l'INSERM et à la Commission européenne. Les deux coauteurs partagent en effet une même démarche d'épistémologie critique ${ }^{2}$. Croiser à l'occasion l'examen de cet ouvrage collectif avec une lecture vagabonde du considérable essai où, sur la longue durée du corpus philosophique, Élisabeth de Fontenay met la métaphysique humaniste à l'épreuve de l'animalité, m’a semblé éclairant.

Un « comment ça se mesure? » résume l'attitude des scientifiques - chercheurs, ingénieurs, techniciens - chargés d'évaluer en termes de bien-être les réactions d'une espèce animale aux conditions de l'élevage confiné. On estime par consensus à l'INRA que le bien-être d'un animal de rente sera satisfait, d'abord s'il ne présente ni blessures ni symptômes de maladie (pathologie), ensuite si ses capacités d'adaptation ne sont pas sollicitées exagérément (physiologie), enfin s'il ne manifeste pas de symptômes d'ennui ou de frustration (comportement). Disons qu'il s'agit d'une définition négative du bien-être. En effet, le présupposé implicite est qu'une interrogation sur la possible incompatibilité des systèmes d'élevage intensif confiné avec la notion même de bien-être serait hors-sujet. L'objectif sera d'adapter les espèces animales à la violence structurelle desdits systèmes. Il y a donc identification entre bien-être et adaptation. Le consensus sur la notion d'adaptation

2. Florence Burgat, «Bien-être animal : la réponse des scientifiques» (pp. 105-133) et Robert Dantzer, "Comment les recherches sur la biologie du bien-être animal se sont-elles construites ? (pp. 85-104), ainsi que l'introduction des coauteurs. L'INRA et divers instituts techniques: le Centre national d'études vétérinaires et alimentaires (CNEVA), l'Institut de l'élevage, l'Institut technique de l'aviculture (ITAVI), l'Institut technique du porc (ITP) et le Comité scientifique (européen) de la santé et de la protection animales, constituent leur terrain d'enquête, de même que le corpus des publications des dernières décennies sur le thème. 
établi, reste le choix des indicateurs. Les indicateurs physiologiques sont ceux qui répondent le mieux à un idéal de réduction de l'objet vivant au quantifiable. La méthode relève de la théorie du stress, terme emprunté à la physique des matériaux (déformation voire rupture sous une contrainte, ou retour à l'état initial quand elle cesse). Le matériau biologique sur lequel s'exercent les contraintes est défini par l'ensemble des processus qui régulent la constance du milieu intérieur d'un organisme (l'homéostasie). Les contraintes du milieu environnant deviennent-elles trop fortes, l'organisme a une réaction de stress (syndrome général d'adaptation). Dans sa formulation originale, la théorie du stress suppose un couplage linéaire entre un événement extérieur traumatisant et une réaction physiologique de l'organisme, sans qu'intervienne aucune médiation de l'appareil mental. Passif, l'animal n'existe que par l'automatisme de ses réactions organiques (sécrétions hormonales, etc.). Ce réductionnisme physiologique de type behavioriste est une position théorique difficile à tenir, d'autant que l'interprétation des indicateurs physiologiques s'est compliquée par suite même des progrès en biologie. La tendance est de les croiser avec l'observation du comportement.

L'éthologie est, à l'origine, la science des comportements des espèces animales dans leur milieu naturel. Les bêtes domestiques vivent dans un milieu façonné par l'homme pour les contrôler. La question est de percevoir jusqu'où l'environnement construit par tel système domesticatoire permet à l'individu d'exprimer le comportement inscrit dans le patrimoine génétique de l'espèce. Pour une poule, ce sera par exemple de picorer et de gratter le sol, de voleter, de prendre des bains de poussière (soin du plumage). Le danger est d'hypertrophier le déterminisme génétique du comportement animal. Qu'en est-il des interactions dans un groupe hiérarchisé de congénères, dont sont d'ailleurs privés les animaux de batterie? Dans les années 1920, l'éthologue Jakob von Uexküll pose que la découpe, chez les animaux supérieurs, d'un environnement extérieur structuré en fonction des besoins de l'espèce (Umwelt) avait son correspondant dans une représentation intériorisée avec sa temporalité propre (Gegenwelt). Cette avancée éthologique a permis au philosophe Merleau-Ponty d'entreprendre une réflexion phénomènologique, en désaccord avec celle de Heidegger, sur une possible ouverture à un être-au-monde de l'être-dans-lemonde de l'animal - pour le dire vite ${ }^{3}$. Ces dernières décennies, les éthologues de terrain ont avancé la notion de "culture animale " pour désigner la transmission stabilisée au sein du groupe de comportements nouveaux acquis par tel ou tel individu. De leur côté, les agronomes zootechniciens qui étudient le comportement des animaux de rente dans le milieu entièrement artificialisé des élevages hors-sol se réferent en principe à la théorie du coping. Formulée par des psychologues, celle-ci traite de l'adaptation psychologique (to cope: se débrouiller, to cope with: affronter). On raisonne en termes de représentation mentale que l'individu se fait de l'événement, de stratégie d'ajustement à une situation, de potentiel cognitif, de subjectivité. Mais comment pénétrer jusqu’à l'intériorité de la brute muette enserrée dans l'appareillage de confinement et de contention?

3. Maurice Merleau-Ponty, La Nature. Dominique Séglard, ed., Paris, Seuil, 1995. L'interprétation de la problématique heideggérienne a donné lieu à controverses. 
"Parle et je te baptise! ", aurait un jour déclaré, d'après Diderot, le cardinal de Polignac à un grand singe du jardin du Roi dans sa cage de verre ${ }^{4}$. Désormais l'animal de rente passé au laboratoire est sommé plus trivialement: "Choisis! clé verte ou clé rouge? la cage élargie ou la double ration?" Sans trop réfléchir sur leurs méthodes, des expérimentateurs rivalisent pour concevoir d'ingénieux dispositifs afin de tester les préférences d'animaux un moment assimilés à des consommateurs, avant d'être eux-mêmes consommés. La coïncidence pourtant demeure hasardeuse entre le dispositif imaginé par l'homme et l'univers subjectif de l'animal. Un moraliste américain relevant de la tradition de la philosophie analytique,Thomas Nagel, s'est confronté à la difficulté d'accéder pour l'homme à l'expérience d'une autre espèce animale ${ }^{5}$. Il s'est demandé l'impression que cela pourrait faire d'être une chauve-souris, mammifere supérieur dont le système perceptif consiste en un sonar (radar fonctionnant par écho) et qui passe la journée dans l'obscurité suspendu par les pattes, la tête en bas. Seule l'imagination, pour Nagel, permettrait de transcender les barrières entre les espèces. Moins imaginatifs que le philosophe, les zootechniciens qui se réclament, pour étudier le bien-être animal, de la théorie du coping en ont trop souvent une conception étriquée, segmentant l'observation du comportement en examen de postures isolées : comment l'animal prend-il sa nourriture, se couche-t-il et se relève, etc. La sélection des séquences d'observation ne fait que reproduire la structure du système confiné et de ses contraintes. Les stéréotypies - une séquence de mouvements à peine ébauchés exprimés de façon répétée et sans utilité apparente (p. 94) - seront interprétées en termes d'adaptation. Prenons les truies en élevage intensif, portées à mordiller de façon compulsive jusqu'à des heures durant soit leur chaîne ou un autre objet, soit à vide. On estime généralement que l'animal alimenté par un dispositif automatique compense de cette manière l'activité normale de quête de nourriture dans un environnement naturel, gratter et fouiller chez le porc. Des chercheurs jugent que cette conduite d'apparence anormale n'aurait rien de pathologique - le profane pense la bête rendue folle - et ne serait qu'une forme d'adaptation à un environnement trop peu stimulant. La stéréotypie de la truie est supposée par certains enclencher la production d'endorphines dans le cerveau. La fragmentation du comportement en postures isolées par l'observateur revient à éliminer la temporalité unifiante de la subjectivité de l'individu, et donc la capacité de l'animal à éprouver des émotions par anticipation. Il existe une définition des besoins comportementaux en termes d'adaptation relevant d'une approche ergonomique. L'animal de rente est considéré alors comme un travailleur d'un type spécial, "un être qui se produit lui-même comme produit » (p. 174), soit six mois pour le porc standard de $105 \mathrm{~kg}$. L'approche ergonomique conséquente ne se limitera pas à l'examen de postures isolées mais étudiera les " comportements organisés » (p. 98). On expérimente ainsi que des porcelets qui, à cause de l'exiguïté ou du sol glissant de leur cage, n'ont pu pratiquer des jeux agonistiques (simulacres de lutte, etc.), seront par la suite beaucoup plus agressifs avec des congénères inconnus lors des transports et des regroupements, et donc plus difficilement manipulables.

4. Élisabeth de Fontenay, pp. 329, 332, 382, 430, 431.

5. Thomas Nagel, Questions mortelles, Paris, PUF, 1983, cité par Élisabeth de Fontenay pp. 693-694. 
Interrogés, les chercheurs qui expérimentent sur le bien-être animal estiment répondre à la "demande sociale» - terme du jargon des appels d'offre - qu'exprime une opinion insatisfaite de la manière dont sont traitées les animaux de rente. La demande reste floue à leurs yeux car médiatisée par l'employeur, à savoir des institutions dont la priorité demeure la productivité économique. En logique comptable, l'élevage en batterie permet de produire au moindre prix des protéines d'origine animale, et le scientifique se défausse volontiers sur le consommateur, sommé d'accepter le renchérissement des coûts lié à des conditions d'élevage plus respectueuses de l'animal. On pourrait objecter qu'il s'agit d'une comptabilité falsifiée qui externalise aux dépens de la collectivité le coût des dommages écologiques afférents (pollution des nappes phréatiques par les lisiers notamment), moins d'ailleurs au bénéfice des producteurs individuels qu'à celui des entreprises industrielles et commerciales qui, par des biais divers, intègrent les filières de production. En a-t-il conscience, il reste hasardeux pour un chercheur d'opposer sa perception globale des enjeux sociétaux au dogme économique obstinément parcellaire de l'institut qui le salarie. Il peut de même n'être pas indifférent au choc émotionnel de la souffrance animale que lui inflige la fréquentation des sites d'élevage hors-sol, mais les affects sont censés par principe ne pas devoir intervenir dans la construction de l'objet d'étude. Un chercheur à l'INRA, Étienne Landais, observe que l'autocensure débouche souvent sur une "pratique schizophrénique » où le scientifique réprouve à titre personnel les effets des procédés qu'il développe en tant que professionnel (p. 124).

La croyance en la seule finalité économique des animaux d'élevage, leur réduction au statut de simple matière première renvoient à un préjugé propre à la société industrielle. La catégorie de l'économique elle-même est une notion moderne dont on suit l'émergence. Élisabeth de Fontenay nous donne les éléments d'une généalogie par référence au corpus philosophique. Débattre de la légitimité de la nourriture carnée était dans l'Antiquité un topos. Que pensait l'homme qui le premier fit «sa nourriture de membres d'animaux qui peu auparavant bêlaient, mugissaient, marchaient et voyaient? Comment ses yeux purent-ils souffrir de voir un meurtre? De voir tuer ? Écorcher, démembrer une pauvre bête? [...] Recevoir le sang et le suc sortant des plaies mortelles d'autrui ? " ${ }^{6}$. L'abstinence de chair animale est motivée différemment selon les écoles antiques. Il peut s'agir d'une simple frugalité visant à la maitrise de soi, d'accès à une vie plus spirituelle, d'exigence de pureté religieuse; l'animal en tant qu'un autrui vivant n'est alors pas concerné. C'est en effet la consommation de sa chair, non le meurtre, qui importait aux cyniques, aux stoïciens, aux épicuriens. Par contre, la pitié à l'égard de l'animal, souvent liée à une croyance en la migration des âmes, est prônée chez les présocratiques par Pythagore et Empédocle, développée ensuite par Théophraste, le successeur d'Aristote au Lycée, puis plusieurs siècles après par Plutarque, enfin reprise par Porphyre, le dis-

6. S'il est loisible de manger chair, in Plutarque, traduit par Amyot, Trois traités pour les animaux, préface d'Élisabeth de Fontenay, Paris, POL, 1992: 105. 
ciple de Plotin ${ }^{7}$. Notre appréhension des textes philosophiques canoniques a été renouvelée ces dernières décennies par les travaux d'hellénistes anthropologues qui nous permettent de mieux saisir les enjeux. Pour Aristote, l'animal, sensible et doué de mémoire certes, est un alogon privé à la fois de la parole et de la raison (polysémie du mot logos), alors que l'usage de la parole, activité humaine fondatrice, entraîne la définition de l'homme comme animal politique. Mais les bêtes domestiques ont participé au politique par le biais des pratiques sacrificielles de la cité antique. Le schéma est celui d'un groupe humain qui se constitue symboliquement comme tel en sacrifiant un animal à une divinité selon un rituel, et en le consommant ensuite collectivement. La victime animale occupe une place éminente dans la configuration triangulaire ainsi formée car elle est une médiation entre les hommes et les dieux. Grillée ou bouillie - consommer de la viande crue est une transgression analogue au cannibalisme -, la chair revient aux hommes, la fumée des os brûlés aux dieux. Le rituel du sacrifice alimentaire s'organise en tant que don, c'est une relation d'échange centrée sur l'animal. Le sacrifice du bœuf laboureur, la bête domestique bienfaisante par excellence, faisait problème au point de simuler un consentement de l'animal à son propre sacrifice (lui faire hocher la tête par quelque ruse...); une cérémonie expiatoire, les Bouphonies, existait à Athènes. Prôner le végétarisme avait des implications politiques sérieuses puisque la condamnation du sacrifice animal mettait en cause un rituel civique fondamental. Avec l'avènement du christianisme la triangularité sacrificielle sera bouleversée. La seule victime désormais admise est le Christ, un dieu fait homme, dont on commémore rituellement le sacrifice accompli en un temps historiquement situé. La bête sacrificielle est radicalement expulsée de la relation au divin (l'assimilation du Christ à l'agneau pascal n'est qu'une métaphore) ${ }^{8}$. Saint Augustin boucle le processus d'expulsion de l'animal du nouveau système symbolique religieux qui se met en place avec l'institutionnalisation de l'Église. Dieu a créé l'homme comme individu doué d'un animus immortel, argumente-il, alors que les animaux, pourvus d'une simple anima, âme sensitive mortelle - Augustin reste vitaliste à la façon d'Aristote - n'ont été créés qu'en tant qu'espèces. Le Christ n’a ainsi racheté par sa mort que les seules créatures humaines. La souffrance des bêtes est théologiquement évacuée grâce à une argutie syllogistique: sous un Dieu juste personne ne peut souffrir à moins d'avoir péché, or les bêtes sont innocentes, donc elles ne souffrent pas. Au final nous n'aurions avec les animaux pas plus de société que nous n'en avons avec les plantes. Thomas d'Aquin, s'il recommande la douceur à l'égard des bêtes, renchérira en les dépossédant de leur anima aristotélicienne. Les brebis silhouettées par Dante dans les vers cités en exergue évoquent une gente d'anime, une troupe d'âmes "qui s’avançaient vers nous, sans qu’il parut, tant elles étaient lentes " (III, 57-60), lenteur qui me semble une dénégation allusive par le poète de l'appauvrissement de

7. Cette liste pour permettre au lecteur de se repérer, grâce à l'index, dans le vaste dédale de l'essai d'Élisabeth de Fontenay, dont le mode d'exposition n'est pas celui d'un manuel d'histoire de la philosophie. 8. L'auteur se démarque (pp. 248-249) de la thèse sacrificielle de René Girard en s'appuyant, entre autres, sur l'argumentation de Marcel Detienne et Jean-Pierre Vernant développée dans La Cuisine du sacrifice en pays grec, Paris, Gallimard, 1972 ("Bibliothèque des Histoires »). 
l'animalité par le théologien. Élisabeth de Fontenay pose que le rapide abandon de la pratique sacrificielle dans l'Occident chrétien a peu à peu conduit à une " appropriation sans foi ni loi de la nature y compris de ceux [les animaux] qui, en elle, s'apparentent plus à l'humain qu'au végétal. Le sang et l'âme, ce lien entre les vivants qui rendait possible les substitutions - âme pour âme, âme contre âme -, tout cela a disparu, et la souffrance animale n'a plus aucune signification puisqu'elle n'a pas été comptée dans le rachat [christique] et que les bêtes sont de moins en moins animées et donc animales, qu'elles ont perdu leur destin d'âmes» (p. 250). La thèse, forte, n'implique pas de crispation passéiste de la part de la philosophe, qui se garde d'accorder une quelconque dimension de sacré sacrificiel à la pratique moderne de la chasse à courre ou de la corrida, mais une inquiétude civilisationnelle au tournant d'un siècle placé sous le signe du clonage. La sélection empirique à l'origine des "races" (variétés) domestiques anciennes a désormais cédé la place aux manipulations biotechnologiques, qu'avait inaugurées l'insémination artificielle. Les modifications génétiques permettent déjà de fabriquer de véritables chimères au sens zoologique du terme.

En dehors de celui d'être dévoré, les animaux de rente peuvent-ils avoir des droits? Dans leTraité de l'abstinence, Porphyre résume, sans en être d'accord, une opinion commune à diverses écoles philosophiques antiques: le fait d'étendre aux animaux le droit détruit le droit ${ }^{9}$. À l'époque moderne, la formulation par l'humanisme juridique d'un lieu commun analogue sera que l'animal ne peut être sujet mais seulement objet de droit, car par définition il ne peut être partie prenante dans un contrat comme sujet autonome et raisonnable ${ }^{10}$. Nous sommes sous le fil de «la différence anthropologique", d'une coupure métaphysique qui tranche radicalement entre l'Être de l'être humain et l'animalité. Les hommes auront dans ce contexte au mieux des devoirs envers les animaux. Le législateur s'est pourtant trouvé dans la société industrielle peu à peu amené à promulguer sous la pression des associations de protection un ensemble de mesures protectrices assez disparates mais qui font masse et posent à nouveaux frais la possibilité d'un droit des animaux ${ }^{11}$. Le débat se déplace alors vers les médiations nécessaires pour qu’un sujet non autonome accède au plan du droit. Joël Feinberg, philosophe américain, développe ainsi une théorie subtile des droits-titres et de la représentation des animaux. Un droit-titre est celui que l'on possède contre quelqu'un, tel le droit du créancier sur son débiteur. La réglementation interdisant la cruauté envers les animaux leur confere un droittitre à en être protégé. L'animal étant incapable de revendiquer ce droit, il lui faut être représenté. Il le sera comme l'est un adulte mis sous tutelle, et ainsi se trouvera titulaire d'un droit. On peut dire aussi que l'animal, incapable de déterminer ses intérêts, a pourtant la capacité de bénéficier des actions des autres, ce qui lui confere un droit, analogue à celui par exemple du droit à l'éducation de l'enfant, qui n'est

9. Élisabeh de Fontenay, p. 182.

10. Jean-Yves Goffi, «L'utilitarisme, les droits et le bien-être animal» (pp. 149-163).

11. Florence Burgat, "Les revendications des associations de protection des animaux d'élevage" (pp. 65-84). 
pas non plus en état de le formuler. Pour définir en général la nature des intérêts des animaux, on les rattachera à la vie conitive (conatus: "tentative»), à la tendance de tout être vivant à persévérer dans son être. Raisonnant en termes d'intérêts, Feinberg se place à mi-chemin entre la tradition utilitariste et l'humanisme juridique comme ce dernier, il refuse à l'animal d'être sujet de droit, mais lui accorde par contre d'avoir des droits. L'utilitarisme, fondé par Jeremy Bentham - on connait l'apostrophe: "la question n'est pas : peuvent-ils [les animaux] raisonner ? ni : peuvent-ils parler ? mais : peuvent-ils souffrir ? "-, argumente en termes d'intérêt de l'individu, de choix des priorité entre catégories d'intérêts, en référence au plus grand bonheur du plus grand nombre. Peter Singer en est sur le thème un représentant contemporain notable. Tout jugement moral ayant un caractère d'obligation et d'universalité ( «l'universalisabilité » de R. M. Hare, dont Peter Singer fut un étudiant à Oxford), le principe d'impartialité impose de ne pas se limiter à l'espèce humaine - ce qui serait de l'espécisme, de même qu'il y a racisme ou sexisme - et d'inclure tous les êtres sensibles capables de souffrir de notre fait dans l'espace moral. Feinberg et Singer argumentent de façon fort différente mais se rejoignent en ce qu'ils reconnaissent aux animaux supérieurs, mammiferes et oiseaux, dont relèvent la plupart des animaux de rente, une capacité à bénéficier des protections normatives définies pour les êtres humains.

Le soupçon d'anthropomorphisme est censé disqualifier un discours sur l'ani$\mathrm{mal}^{12}$. J'examinerai la notion à partir d'un cas littéraire : l'influence sur Montaigne d'un apologue du Discours de la servitude volontaire, pamphlet d'une cinquantaine de pages écrit par La Boétie adolescent vers 1549. Pourquoi, s'interroge-t-il, lorsque les hommes se constituent en corps politique, le font-ils en général sous forme de complète aliénation de leur liberté entre les mains d'un seul ? Question iconoclaste, les monarques sont absolus de droit divin. L'animalité y est mise en scène dans un passage dont je résume l'argument. Il s'agit de rendre la multitude honteuse de son asservissement à un seul, qui ne vaut pas plus que chacun, voire le pire d'entre eux, et de lui rappeler que la liberté est naturelle. On convoque alors par manière de dire les bestes brutes en chaire, pour vous enseigner votre nature et condition. Les bestes, ce m'aid'Dieu, si les hommes ne font trop les sourds, leur crient, vive liberté. Capturées, certaines se laisseront périr : Si les animaux avoient entre eux leurs rangs et quelques prééminences, ils feraient (à mon avis) de liberté leur noblesse. Les espèces domestiques, destinées pourtant au service de l'homme, ne manquent aussi de résister: Nous appâtons le cheval dès lors quill est né, pour l'apprivoiser à servir; et si ne le savonsnous tant flatter que quand ce vient à le dompter il ne morde le frein, quil ne rue contre l'éperon, comme, ce semble, pour montrer à la nature, et témoigner au moins par là que sil sert, ce n'est pas de son gré, mais par notre contrainte. Que faut-il donc dire? Mêmes les boufs sous le poids du joug geignent. Et les oiseaux dans la caige se pleignent. Le désir d'être asservi demeure inconnu des animaux, en quoi l'espèce humaine leur est devenue inférieure. En conclusion, l'interrogation est réitérée sur ce malencontre

12. Françoise Armengaud, "L'anthropomorphisme : vraie question ou faux débat ?" (pp. 165-187). 
qui a pu tant dénaturer l'homme, jusquà lui faire perdre la souvenance de son premier être, et le désir de le reprendre ${ }^{13}$. Le mot clé est "dénaturer". Montaigne a adhéré aux vues de l'ami disparu prématurément auquel il s'identifia en son projet d'écrivain, seul le contexte politique des guerres de religion l'empêche d'insérer le Discours au cœur de la première édition des Essais (1580). Les hommes en société lui apparaissent comme des animaux dénaturés à qui il faut une coustume: lois, mythes, institutions, croyances religieuses, sans que l'on puisse déduire de cette nécessité empirique de la vie en société une quelconque identité de la coustume avec la raison. Des peuples du Nouveau Monde avaient une coustume plus proche de la nature, nous les avons détruits à peine découverts. L'arbitraire de la coustume, inhérent à l'être social, semble contredire à la fois le cours de la nature et l'exercice de la raison, paradoxe désespéré. Reconsidérons la mise en scène des animaux dans l'apologue de La Boétie, formellement décomposable en une série d'exempla puisés dans le corpus gréco-latin, voire dans les traditions populaires du Carnaval et du monde à l'envers. La violence rhétorique de la comparaison ainsi établie des hommes avec les bêtes n'est pas sans effets sur le lecteur. Aux humains que la recherche de l'unité avec leurs semblables, dévoyée en désir d'asservissement, ont réduit à consentir à devenir l'objet d'un maitre est proposé un scandaleux miroir : les bêtes. Simples objets pour la satisfaction de nos besoins, leur désir inextinguible de liberté en fait l'image du libre sujet humain perdu. On semble revenu au temps où les animaux parlaient, mais nous sommes ici moins dans la temporalité du conte que dans un espace rhétorique. $\mathrm{Si}$ on adopte le principe selon lequel la syntaxe serait le point de départ de la rhétorique ${ }^{14}$, il y a, quant à la syntaxe du récit, échange de position entre les termes, puisque face aux hommes réduits par euxmêmes à l'état d'objet les animaux occupent la figure du sujet. C'est façon de dire, objectera-t-on, l'intention de La Boétie a été de faire honte aux humains, non de s'occuper du sort des bêtes, ni des relations établies avec elles, encore moins de les poser en sujet. Il existe pourtant une logique de la fiction, la description d'une aussi farouche rébellion n'est pas sans éveiller chez le lecteur des échos utopiques. Par un écart de sens on passe de la violence, d'ordre politique, que s'infligent les hommes, à celle qu'il font subir aux autres êtres vivants. De quel droit, esclaves complices de notre asservissement, opprimer des êtres qui se veulent libres? La leçon qu'ils donnent ne mérite-elle pas de notre part à tout le moins quelque respect envers eux ? etc. L'implacable argumentaire du Discours finit par produire un effet fictionnel en faveur des bêtes. Identifier l'ambivalence de l'espace rhétorique construit par La Boétie nous permet d'imaginer le contexte réflexif dans lequel se meut l'ami survivant qui prend en considération les animaux, condamne la cruauté à leur égard, élabore une relation d'ordre axiologique aux corps vivants périssables, sujets à la jouissance et à la souffrance, de l'être humain aux bêtes, voire au végétal. L'auteur

13. Étienne de La Boétie, Le Discours de la servitude volontaire, Paris, Payot, 1976 : 120-122. Orthographe modernisée, sauf quand la graphie ancienne nous a paru garder une valeur expressive. Je développe ici partie d'une communication, "Descartes lecteur de Montaigne. Quel statut pour l'animalité ? ", faite au colloque de l'INRA ayant pour thème «Bien-être animal et comportement humain » (Paris, 6-7 nov. 1995).

14. Mitsou Ronat, Change de forme, Paris, 1975 («10/18») : 300. 
des Essais avait l'expérience du quotidien d'un domaine rural et du maniement des chevaux commune à la petite noblesse provinciale.

Le marketing publicitaire se montre soucieux de réenchanter notre rapport aux bêtes à viande. Notre propos est plutôt de développer à leur endroit une approche réflexive. La relation éleveur-animal constitue d'un point de vue éthologique un phénomène d'interface, au cœur du processus historique de domestication. Elle met en jeu une adaptation mutuelle au comportement de l'autre qui entrâne au quotidien un échange d'affects, un compagnonnage comportemental ${ }^{15}$ défait sans recours par l'industrialisation de l'élevage. Comme l'enquête de Jocelyne Porcher l'établit, la mise en place du système intensif a partie liée avec une occultation volontaire de l'affectivité de l'éleveur envers ses bêtes ${ }^{16}$. Le collectif familial d'une exploitation qui a en charge un élevage hors-sol, sommé de se conformer à l'impératif d'efficacité du modèle industriel, intériorise la nécessité de "la chasse aux temps improductifs", justement ceux où peut se créer une relation désintéressée avec l'animal (pp. 41-42). Le bien-être d'un animal, puisque c'est de cela qu'il s'agit, dépend en partie du plaisir que prend à l'élever celui qui en a la charge. A contrario, la violence structurelle de l'élevage intensif induit une contagion de la souffrance entre hommes et bêtes. En consentant à la souffrance que sa pratique inflige à l'animal, l'éleveur consent à sa propre souffrance, celle qu'il subit du fait de sa sujétion aux normes industrielles. On généralisera en disant que la réification de l'animal induite par l'élevage industriel déshumanise l'éleveur, au même titre que le chercheur zootechnicien dont nous avons déjà repéré l'aliénation ${ }^{17}$. Qu'en est-il du consommateur, les mangeurs de viande que nous sommes? La mise à mort des animaux est depuis longtemps occultée dans des abattoirs rejetés vers les périphéries. Il n'y a rien à voir. Par un processus analogue d'occultation, les mauvais traitements infligés aux animaux et le profond mal-être des éleveurs qu'induit l'élevage industriel, mis hors-vue dans les hangars du hors-sol, sont déréalisés aux yeux d'un consommateur guère désireux de trop en savoir, ce que nous synthétisions en ouverture par l'image de la viande sous cellophane ${ }^{18}$. Promouvoir effectivement le bien-être des animaux de rente demande que la recherche zootechnique institutionnelle accepte de prendre en compte les phénomènes de refoulement des affects qui caractérisent le système d'élevage indus-

15. Je préfere cette expression à la métaphore juridique employée par Catherine et Raphaël Larrère, «L'animal, machine à produire : la rupture du contrat domestique » (pp. 9-24), dont je discerne mal la valeur heuristique. Le cavalier et sa monture offrent un cas de figure achevé de compagnonnage comportemental. 16. Jocelyne Porcher, «Le travail dans l'élevage industriel des porcs. Souffrance des animaux, souffrance des hommes" (pp. 25-64).

17. En témoignent, exemple parmi d'autres, les réactions des éleveurs à la manière dont les pouvoirs publics traitèrent une épidémie de fièvre aphteuse (automne 2000 à mi-2001), préférant, pour des raisons commerciales semble-t-il, à la vaccination des bêtes l'abattage "préventif " par dizaines de milliers (Le Monde, 31/3/01). Les systèmes d'élevage intensif confiné ne sont pas les seuls concernés (cf. n. 18). 18. On sait que les téléspectateurs - avatars du "consommateur» - ont été choqués par le spectacle de bûchers d'incinération de carcasses de troupeaux victimes des épidémies d'encéphalite spongiforme bovine (ESB), de fièvre aphteuse, etc. Rappelons que, par le biais des compléments alimentaires pour bétail (farines animales, facteur de l'ESB) et de la circulation des bêtes sur pied dans le contexte commercial européen (contagion), les bûchers concernent autant l'élevage industriel de type extensif ou semi-extensif que les systèmes intensifs confinés, objet du présent article. La relation au bétail d'embouche - «on n'a plus le temps de s'en occuper ", formule devenue un leitmotiv - ressortit à une problématique éthologique qu'il n’y a pas lieu de développer ici. 
triel sous ses formes diverses. L'édition par l'INRA de l'ouvrage recensé semble aller dans ce sens, mais l'absence de réaction à sa publication de la part du milieu d'abord concerné, la recherche zootechnique, laisse perplexe. Une autre hypothèse serait que la préoccupation affichée du bien-être animal vise un simple effet d'annonce à l'intention d'une opinion publique dépourvue de repères, ce que l'on appelle, en termes de marketing, " communiquer».

MOTS CLÉS/KEYWORDS : animalitélanimality - animaux de rente/farm animals - animal sacrifi$\mathrm{ciel} /$ sacricial animal - bien-être animal/animal welfare - recherche zootechnique/zootechnical research. 
